\title{
The Long Non-Coding RNA XIST Interacted with MiR-124 to Modulate Bladder Cancer Growth, Invasion and Migration by Targeting Androgen Receptor (AR)
}

\author{
Yaoyao Xiong ${ }^{\mathrm{b}}$ Long Wang ${ }^{\mathrm{a}} \quad$ Yuan Li ${ }^{\mathrm{a}}$ Minfeng Chen ${ }^{\mathrm{a}}$ Wei He $\mathrm{Lin}^{\mathrm{a}} \mathrm{i}^{\mathrm{a}}$ \\ aDepartment of Urology, Xiangya Hospital, Central South University, Changsha, Hunan, bepartment \\ of Cardiopulmonary Bypass, The Second Xiangya Hospital, Central South University, Changsha, Hunan, \\ China
}

\section{Key Words}

Xist • MiR-124 • Bladder cancer • Cell proliferation • Invasion • Migration • Androgen receptor $(A R)$

\begin{abstract}
Backgrounds/Aims: Long non-coding RNA (IncRNA) X-inactive specific transcript (XIST) is involved in the progression of several tumors. The interaction between IncRNA and miRNA or miRNA's target genes is reported to play crucial roles in malignancy. In addition, Androgen receptor (AR) is considered to be involved in bladder cancer progression. In this study, we investigated the role of XIST in human bladder cancer and its interaction with miR-124 and AR. Methods: XIST and AR expression was detected in bladder tumor samples and cell lines. Effects of XIST and AR on bladder cancer cells growth, invasion and migration were analyzed. Bioinformatic analysis and luciferase assays were used to identify the interaction among XIST, AR and miR-124. The correlations of miR-124 with XIST and AR in bladder cancer samples were statistically analyzed. Results: XIST and AR were upregulated in bladder cancer tissues and positively correlated. Higher XIST and AR expression were related to poorer TNM stage of bladder cancer. XIST knockdown reduced bladder cancer cells' proliferation, invasion and migration. While this inhibitory effect could be partially restored by AR overexpression. XIST inhibited miR-124 expression by directly targeting. Moreover, miR-124 could bind to the $3^{\prime} U T R$ of AR to regulate its expression. MiR-124 inhibition partially restored the XIST knockdown-induced reduction of AR, c-myc, p27, MMP13 and MMP9 expression. In bladder cancer tissues, miR-124 level was inversely correlated with the expression of XIST and AR, respectively. Conclusion: These findings indicated that XIST might be an oncogenic IncRNA that promoted the bladder cancer growth, invasion and migration via miR-124 dependent AR regulation.
\end{abstract}




\section{Cellular Physiology Cell Physiol Biochem 2017;43:405-418 \begin{tabular}{l|l|l} 
and Biochemistry Published online: September 01, 2017 & $\begin{array}{l}\text { (c) } 2017 \text { The Author(s). Published by S. Karger AG, Basel } \\
\text { www.karger.com/cpb }\end{array}$
\end{tabular} \\ Xiong et al.: The XIST/miR-124/AR Axis Regulate Bladder Cancer Growth, Invasion and Migration}

\section{Introduction}

Bladder cancer (BC) is the most common and most fatal type of urinary tumor $[1,2]$. Although 5-year survival rates in $\mathrm{BC}$ patients have improved, one third of all patients still experiences recurrence. Emerging evidence suggests that BC recurrence is related to cell proliferation and invasion, and the molecular mechanisms that regulate the proliferation, invasion in $\mathrm{BC}$ are a topic of current research [3-5].

Human genome sequence data indicate that more than $90 \%$ of the DNA sequences actively transcribed but only $2 \%$ of it encodes a protein, thus the majority of transcripts are referred to as non-coding RNAs (ncRNAs) [6, 7]. Small non-coding RNAs such as microRNAs have been studied extensively and their roles in gene regulation and cell function have been elucidated innumerous cancers [7]. Recent studies have shown that long non-coding RNAs (lncRNAs) play important roles in both normal development and diseases including cancer [8]. LncRNAs have emerged as new players in cancer research and several studies has shown that some lncRNAs function as oncogenes, tumor suppressor genes or both, depending on the circumstance [9]. X-inactive specific transcript (XIST), one of the novel-found LncRNA, has been confirmed that dysfunctional expression has a pathological role in cancer [10]. LncRNA XIST can act as a predictive biomarker for screening non-small lung cancer [11]. Moreover, high XIST expression was significantly correlated with gastric cancer tumor size and late TNM stage [12].

The mechanisms by which IncRNAs exert their effect varies under different conditions, however, emerging evidences have revealed that the interaction between lncRNAs and microRNAs plays a major role $[13,14]$. XIST regulates gastric cancer progression by acting as a molecular sponge of miR-101 to modulate EZH2 expression [15]. MiR-92b promotes hepatocellular carcinoma progression by targeting Smad7 and is mediated by XIST [16].

Androgen receptor (AR) is a ligand-dependent transcriptional factor that regulates the biologic functions of androgens. It has been confirmed that AR plays a crucial role in malignancies of the prostate, kidney, breast and bladder cancers [17]. In bladder cancer, high expression of AR has been observed and positively correlated with disease progression [18]. Moreover, AR signaling may also contribute to invasion and metastasis of bladder cancer [17].

In this study, we report an interaction between X-inactive specific transcript (XIST) and miR-124 which regulates bladder cancer cell growth, invasion and migration through directly targeting the androgen receptor (AR). Our findings provide a novel understanding of the role of XIST/miR-124/AR in bladder cancer growth, invasion and migration, and the mechanism involved.

\section{Materials and Methods}

Tissue samples, cell lines

We collected a large panel of 67 paired bladder cancer tissues and the matched adjacent non-tumor tissues. We obtained all samples from patients who underwent surgical resection at Xiangya Hospital of Central South University (Changsha, China). All the human tissues were obtained with informed consent and this study was approved by the Clinical Research Ethics Committee of The Xiangya Hospital of Central South University. The tissues were snap-frozen in liquid nitrogen, and then stored at $-80^{\circ} \mathrm{C}$.

We purchased human bladder cancer cell lines, TCC-SUP, EJ, SW780 and UM-UC-3 cells and human uroepithelium epithelial SV40 immortalized cell SV-HUC-1 from the American Type Culture Collection (Manassas, VA, USA). They were cultured in RPMI-1640 medium (Invitrogen, Carlsbad, CA, USA) supplemented with $10 \%$ fetal bovine serum (Gibco, CA, USA) at $37^{\circ} \mathrm{C}$ in a humidified atmosphere with $5 \%$ $\mathrm{CO}_{2}$.

Cell transfection

The expression of miR-124 was achieved by transfection of miR-124 mimics or miR-124 inhibitor (Genepharma, Shanghai, China) using Lipofectamine 2000 (Invitrogen). A pCMV-AR vector was used to 


\section{Cellular Physiology Cell Physiol Biochem 2017;43:405-418 \\ \begin{tabular}{ll|l}
\hline and Biochemistry 10.1159/000480419 & $\begin{array}{l}\text { C) } 2017 \text { The Author(s). Published by S. Karger AG, Basel } \\
\text { www.karger.com/cpb }\end{array}$ \\
\hline
\end{tabular}}

Xiong et al.: The XIST/miR-124/AR Axis Regulate Bladder Cancer Growth, Invasion and Migration

achieve overexpression of AR (GeneCopoecia, Guangzhou, China). SiRNA1 (sense: 5'-UUU UGA AGC AUA UUU UGG CUU TT-3'; anti-sense: 5'- GCC AAA AUA UGC UUC AAA AGA TT-3') and siRNA2 (sense: 5'-AUA CUU UGG GCC UUC UAU CCA TT-3'; anti-sense: 5'- GAU AGA AGG CCC AAA GUA UAA TT-3')were used to achieve XIST knockdown (GenePharma, Shanghai, China), Cells were plated in 6-well plates or 96-well plates, transfected, incubated for $24 \mathrm{~h}$ or $48 \mathrm{~h}$ and used for further assays or RNA/protein extraction.

\section{RNA extraction and SYBR green quantitative PCR analysis}

We extracted total RNA from cells using Trizol reagent (Invitrogen, CA, USA). For miRNA, RNA was converted into cDNA using miScriptRT kits (Qiagen, German) according to the manufacturer's instruction. Mature miR-124 expressions in cells were detected using a Hairpin-it TM miRNAs qPCR kit (Genepharma, Shanghai, China). We used expression of RNU6B as an endogenous control. For IncRNA and mRNA, RNA was converted in to cDNA using PrimeScript RT kits (Takara, Japan) according to the manufacturer's instruction. The XIST expression and AR mRNA levels was measured by SYBR Green qPCR assay (Takara, Dalian, China). GAPDH was used as endogenous control.Primers were showed in Table 1 .. Data was processed using $2^{-\triangle \Delta C T}$ method.

\section{MTT assay}

A modified MTT assay was used to evaluate cell viability. After seeding $2 \times 10^{3}$ transfected cells/well into 96-well culture plates we assessed the viability of bladder cancer cells transfected with the indicated vectors at five time points (on day 1, 2, 3, 4 and 5). In brief, quantification of mitochondrial dehydrogenase activity was achieved through the enzymatic conversion of MTT [3-(4, 5-dimethyldiazol-2-yl)-2, 5diphenyltetrazolium bromide; Sigma-Aldrich, MO, USA] to a colored formazan product. MTT (10 $\mu$ l, $10 \mathrm{mg} /$ $\mathrm{ml}$ ) was added to the cells, incubated for $4 \mathrm{~h}$, and we terminated the reaction by removal of the supernatant and addition of $100 \mu \mathrm{l}$ DMSO to dissolve the formazan product. After $0.5 \mathrm{~h}$, the optical density (OD) of each well was measured at $450 \mathrm{~nm}$ using a plate reader (ELx808 Bio-Tek Instruments, City, ST, USA).

\section{BrdU assays}

By measuring 5-Bromo-2-deoxyUridine (BrdU) incorporation, the DNA synthesis in proliferating cells was determined. BrdU assays were conducted at $48 \mathrm{~h}$ after TCC-SUP and UM-UC-3 cells were transfected with si-NC/si-Xist and/or pCMV/pCMV-AR vectors. Cells were seeded in 96-well culture plates at a density of $2 \times$ $10^{3}$ cells/well, cultured for $48 \mathrm{~h}$, then incubated with a final concentration of $10 \mu \mathrm{M}$ BrdU (BD Pharmingen, San Diego, CA, USA) for $2 \mathrm{~h}$. When the incubation period ended, the medium was removed, the cells were fixed for $30 \mathrm{~min}$ at RT, incubated with peroxidase-coupled anti-BrdU-antibody (Sigma-Aldrich) for 60 min at RT, washed three times with PBS, incubated with peroxidase substrate (tetramethylbenzidine) for $30 \mathrm{~min}$, and the $490 \mathrm{~nm}$ absorbance values were measured for each well. Background BrdU immunofluorescence was determined in cells not exposed to BrdU but stained with the BrdU antibody.

\section{Transwell assay}

Cells (5× $10^{5}$ cells/well) were plated on the top side of polycarbonate Transwell filter coated(for invasion assays) or uncoated (for migration assays) with Matrigel in the top chamber (Cell Biolabs, Inc. Santiago, USA). Cells were suspended in medium without serum and medium supplemented with serum was used as a chemoattractant in the bottom chamber. The cells were incubated at $37{ }^{\circ} \mathrm{C}$ for $48 \mathrm{~h}$. The noninvasive cells in the top chambers were removed with cotton swabs. The migrated and invaded cells on the lower membrane surface were fixed in $100 \%$ methanol for $10 \mathrm{~min}$, air-dried, then stained with DAPI (Beyotime Institute of Biotechnology, Haimen, China), and counted under a microscope.

\section{Analysis of MMP9 and MMP13 activity}

After transfection for 48h, the activities of MMP9 and MMP13 in culture media were collected and centrifuged at 10, $000 \mathrm{rpm}$ for $5 \mathrm{~min} .25 \mu \mathrm{l}$ of the supernatant was used to measure MMP9 and MMP13 activities by SensoLytes Plus ${ }^{\mathrm{TM}} 520$ MMP9 and MMP13 assay kits (AnaSpec, San Jose, CA, USA) according to the manufacturer's instruction. The fluorescence signal is monitored at Ex/Em=490 nm/520 nm by GloMax®-Multi+ Detection System (Promega, Madison, WI, USA).

Western blot analysis

The expression of AR, c-myc, p27, MMP9 and MMP13 in bladder cancer cells was detected by performing immunoblotting. We lysed cultured or transfected cells in RIPA buffer with $1 \%$ PMSF and loaded protein onto 
a SDS-PAGE minigel and transferred them onto PVDF membrane. After probed with a 1:1000 diluted rabbit polyclonal AR, c-myc, p27, MMP9 and MMP13 antibodies (Santa Cruz, CA, USA) at $4^{\circ} \mathrm{C}$ overnight, the blots were subsequently incubated with HRPconjugated secondary antibody (1:5000). ECL Substrates was used to visualize signals (Millipore, MA, USA). GAPDH was used as an endogenous protein for normalization.

\section{Luciferase reporter assay}

HEK293 cells (ATCC, USA) were seeded into a 24-well plate. For XIST and miR-124 interaction, cells were co-transfected with wild-type, mutated XIST reporter gene plasmid or pRL-TK plasmids, and miR-124 mimics or miR-124 inhibitor. For miR-124 and AR correlation, after cultured overnight, cells were co-transfected with the wildtype, mutated AR 3'UTR reporter plasmid or pRL-TK plasmids, and miR-124 mimics or miR-124 inhibitor. The wild-type or mutant reporter plasmids were purchased from Yrbio tech. Co. Ltd (Changsha, China). Luciferase assays were performed $48 \mathrm{~h}$ after transfection using the Dual Luciferase Reporter Assay System (Promega, WI, USA).

\section{Statistical analysis}

Data were exhibited as mean \pm SD of three independent experiments and processed using SPSS 17.0 statistical software (SPSS, Chicago, IL, USA). By using Student's t-Tests we compared the expression of XIST, AR and miR-124 in bladder cancer tissues and the paired adjacent non-tumor tissues. The differences between groups were evaluated using the one-way ANOVA. The correlation of XIST, miR-124 and AR in BC tissues was analyzed by Spearman's rank correlation analysis. $P$ values of $<0.05$ were considered statistically significant.

\section{Results}

Expression of XIST and AR in bladder cancer tissues and cell lines Here we first determined the expression of XIST and AR in a large panel of 67 bladder cancer tissues and corresponding adjacent non-tumor tissues using real-time PCR. Results showed that both XIST and AR expression was significantly up-

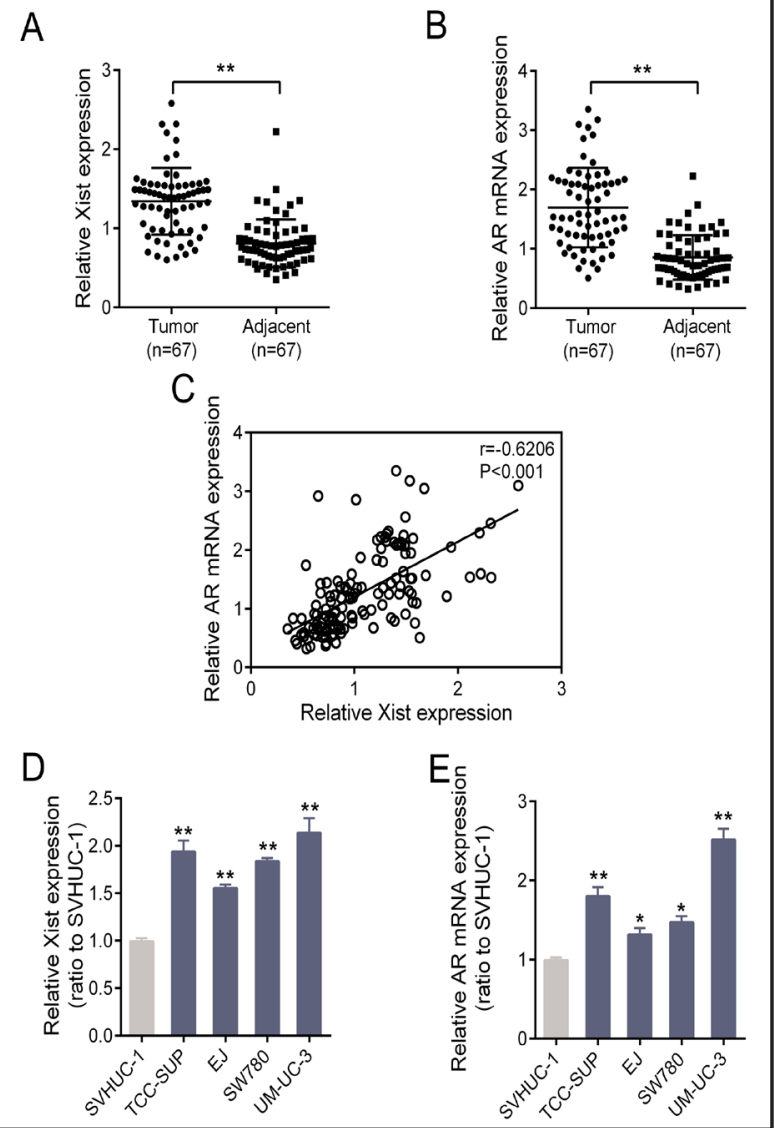

Fig. 1. Expression of XIST and AR in bladder cancer tissues and cell lines (A, B) Expression of XIST and AR in a large panel of 67 paired bladder cancer tissues and corresponding adjacent non-tumor tissues was determined using real-time PCR. (C) The correlation between XIST and AR was analyzed by performing Spearman's rank relation analysis. (D and E) Expression of XIST and AR in four bladder cancer cell lines, TCC-SUP, EJ, SW780 and UM-UC-3, and a normal cell line, SV-HUC-1, was determined using real-time PCR. The data are showed as mean \pm SD of three independent experiments. ${ }^{*} \mathrm{P}<0.05,{ }^{* *} \mathrm{P}<0.01$.

Table 1. Oligonucleotides of real-time PCR

\begin{tabular}{lr} 
Real-time PCR Primer & Sequence \\
\hline MiR-124-F & GTG CAG GGT CCG AGG T \\
MiR-124-R & ATC ATA GAG GAA AAT CCA CG \\
RNU6B -F & CTC GCT TCG GCA GCA CA \\
RNU6B -R & AAC GCT TCA CGA ATT TGC GT \\
XIST-F & CTC TCC ATT GGG TTC CAC \\
XIST-R & GCG GCA GGT CTT AAG AGA TGA G \\
AR-F & CTG GAC ACG ACA ACA ACC AG \\
AR-R & CAG ATC AGG GGC GAA GTA GA \\
GAPDH-F & ACA GGG GAG GTG ATA GCA TT \\
GAPDH-R & GAC CAA AAG CCT TCA TAC ATC TC
\end{tabular}


regulated in tumor tissues compared with the corresponding adjacent non-tumor tissues (Fig. 1A, B). Spearman's rank correlation analysis showed that the expression of XIST and AR was positively correlated in tumor tissues (Fig. 1C). XIST and AR expression in four bladder cancer cell lines, TCC-SUP, EJ, SW780 and UM-UC-3 and a normal cell line, SVHUC-1 was determined using real-time PCR. Results showed that XIST and AR expression was up-regulated in bladder cancer cell lines compared with SV-HUC-1, consistent with the results in bladder cancer tissues (Fig.1D). In addition, to investigate the relation of XIST or AR expression with the clinicopathologic features, we divided 67 patients into two groups, respectively: for XIST, a high XIST expression group (above the mean XIST expression, $\mathrm{n}=$ 34) and a low XIST expression group (below the mean XIST expression, $n=33$ ); for AR, a high AR expression group (above the mean AR expression, $n=34$ ) and a low AR expression group (below the mean AR expression, $\mathrm{n}=33$ ). As exhibited in Table 1, high XIST expression and high AR expression were related to poorer TNM stage of bladder cancer (Table 2).

The role of XIST and AR in bladder cancer cell proliferation, invasion and migration

We demonstrated high expression of XIST and AR in bladder cancer tissues and cell lines, and their relation to poorer TNM stage of bladder cancer; we next investigated the functional role of XIST and AR in bladder cancer proliferation, invasion and migration. Two siRNAs were transfected into TCC-SUP and UM-UC-3 cells to achieve XIST knockdown, and the inhibitory efficiency was verified using real-time PCR assays (Fig. 2A). In both TCCSUP and UM-UC-3 cells, siRNA2 significantly down-regulated XIST expression, and was selected as si-XIST for subsequent experiments (Fig. 2A). TCC-SUP and UM-UC-3 cells were then co-transfected with si-NC/si-XIST and pCMV/AR, and the protein levels of AR were determined using Western blot assays. Results showed that in both TCC-SUP and UM-UC-3 cells, AR protein levels could be reduced by si-XIST transfection and promoted by AR vector transfection, while the inhibitory effect of si-XIST could be partially restored by AR vector transfection (Fig. 2B). After co-transfection, the viability and proliferation of TCC-SUP and UM-UC-3 cells were determined using MTT and BrdU assays. Results showed that the viability and proliferation of TCC-SUP and UM-UC-3 cells was significantly down-regulated by si-XIST transfection, up-regulated by AR overexpression; the inhibitory effect of si-XIST on TCC-SUP and UM-UC-3 cells' viability and proliferation could be partially restored by AR overexpression (Fig. 2C, D). Similar effect of si-XIST was observed in the cell invasion and migration assays: the invasive and migration ability of TCC-SUP and UM-UC-3 cells was down-regulated by si-XIST, up-regulated by AR overexpression, and the inhibitory effect of si-XIST on bladder cancer cells' invasion and migration could be partially restored by AR overexpression (Fig. 2E-F). These data suggested that XIST might affect the cell proliferation and metastasis of bladder cancer through regulating AR expression.

\section{XIST correlated with miR-124 by direct targeting}

We revealed the functional role of XIST and AR in bladder cancer cells' proliferation, invasion and migration; next mechanistic assays were performed to investigate the mechanism

Table 2. Correlation of the expression of Xist and AR with clinicopathologic features

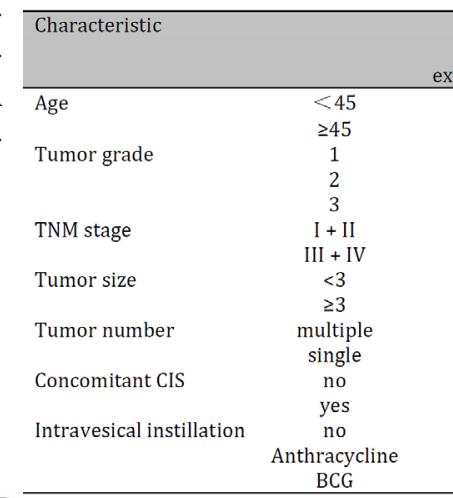

\begin{tabular}{|c|c|c|c|c|c|c|c|c|}
\hline 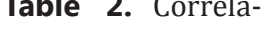 & Characteristic & & Xist ex & ession & $\mathrm{p}$-value & AR exp & ession & p-value \\
\hline tion of the expres- & & & $\begin{array}{l}\text { high } \\
\text { expression } \\
\end{array}$ & $\begin{array}{c}\text { low } \\
\text { expression }\end{array}$ & & $\begin{array}{l}\text { high } \\
\text { expression } \\
\end{array}$ & $\begin{array}{c}\text { low } \\
\text { expression }\end{array}$ & \\
\hline sion of Xist and AR & Age & $<45$ & 14 & 18 & 0.273 & 18 & 14 & 0.389 \\
\hline with cliniconatho- & & $\geq 45$ & 20 & 15 & & 16 & 19 & \\
\hline Witn cimicopacho- & Tumor grade & 1 & 8 & 10 & 0.784 & 9 & 9 & 0.418 \\
\hline logic features & & 2 & 17 & 16 & & 19 & 14 & \\
\hline & & 3 & 9 & 7 & & 6 & 10 & \\
\hline & TNM stage & $\mathrm{I}+\mathrm{II}$ & 14 & 22 & 0.036 & 13 & 23 & 0.010 \\
\hline & & $\mathrm{III}+\mathrm{IV}$ & 20 & 11 & & 21 & 10 & \\
\hline & Tumor size & $<3$ & 13 & 20 & 0.067 & 15 & 18 & 0.393 \\
\hline & & $\geq 3$ & 21 & 13 & & 19 & 15 & \\
\hline & Tumor number & multiple & 17 & 16 & 0.901 & 18 & 15 & 0.540 \\
\hline & & single & 17 & 17 & & 16 & 18 & \\
\hline & Concomitant CIS & no & 26 & 23 & 0.532 & 26 & 23 & 0.532 \\
\hline & & yes & 8 & 10 & & 8 & 10 & \\
\hline & Intravesical instillation & no & 18 & 16 & 0.639 & 21 & 13 & 0.095 \\
\hline & & Anthracycline & 10 & 13 & & 18 & 28 & \\
\hline & & BCG & 6 & 4 & & 12 & 18 & \\
\hline
\end{tabular}




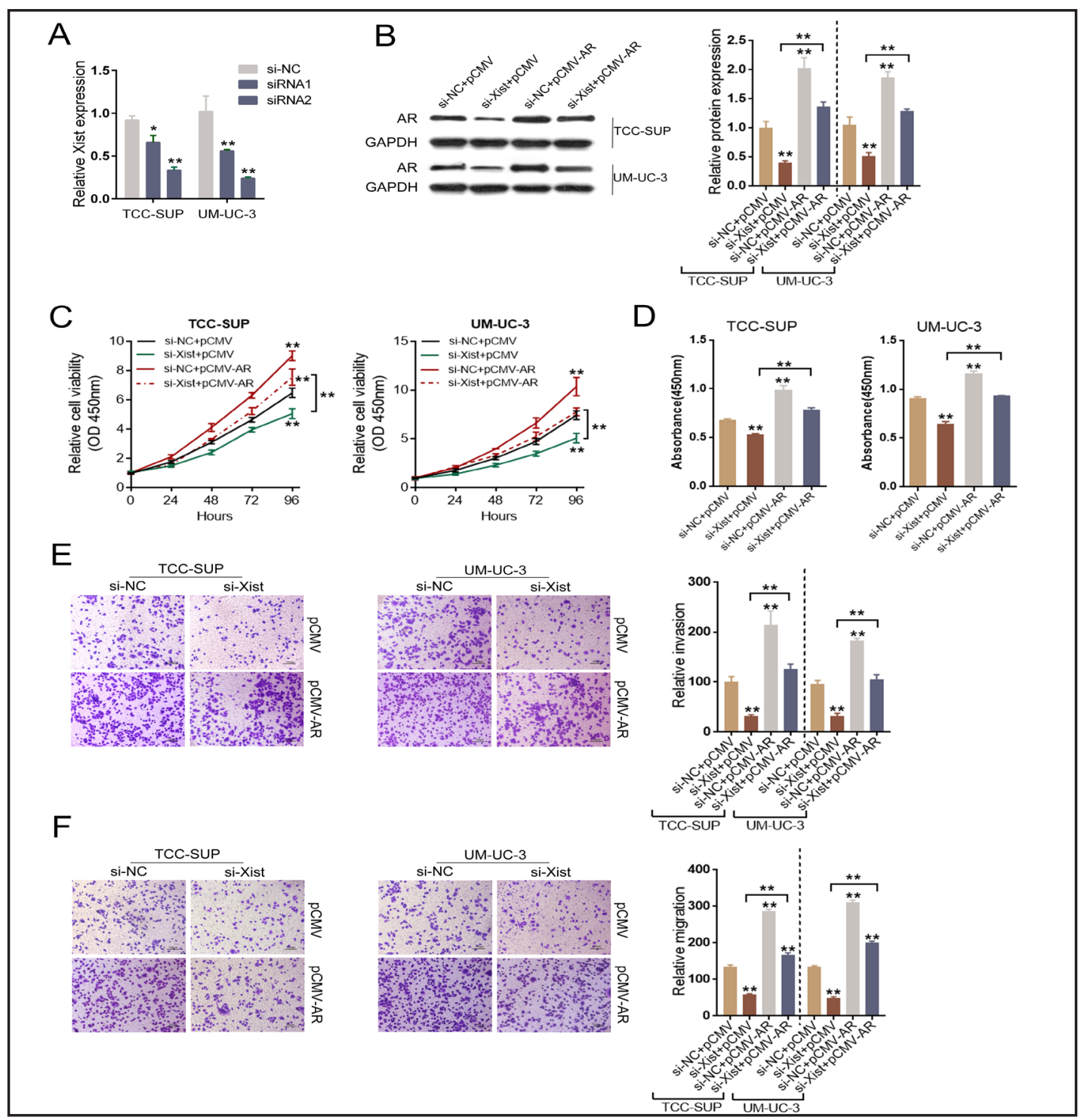

Fig. 2. The detailed role of XIST and AR in bladder cancer cell proliferation, invasion and migration (A) Two siRNAs, siRNA1 and siRNA2 were transfected into TCC-SUP and UM-UC-3 cells to achieve XIST knockdown. The inhibitory efficiency was verified using real-time PCR assays. SiRNA2 was selected as siXIST for the subsequent assays. (B) Si-XIST and pCMV-AR were co-transfected into TCC-SUP and UM-UC-3 cells. Then the protein levels of AR were monitored in each group. Protein level of GAPDH was used as an endogenous normalization. (C, D) MTT assays and BrdU assays were performed to determine the viability and proliferation of TCC-SUP and UM-UC-3 cells after co-transfection with si-NC/si-XIST and pCMV/AR. (E, F) Transwell assays were performed to determine the invasive and migration capability of TCC-SUP and UMUC- 3 cells after co-transfection with si-NC/si-XIST and pCMV/AR. The data are showed as mean \pm SD of three independent experiments. ${ }^{*} \mathrm{P}<0.05,{ }^{*} \mathrm{P}<0.01$.

by which XIST might regulate AR expression. Previous studies have suggested that lncRNAs serve as competing endogenous RNAs (ceRNAs) to sponge miRNAs, thereby regulating gene expression [19]. According to previous studies, miR-124 plays a suppressive role in cancers [20]. The role of miR-124 in bladder cancer was reported [21]. Here, we hypothesized that XIST might act as a ceRNA to sponge miR-124, thus to regulate AR expression. To confirm this hypothesis, miR-124 expression in response to XIST knockdown was monitored using real-time PCR in si-XIST-transfected TCC-SUP and UM-UC-3 cells. Results showed that miR- 
Fig. 3. XIST correlated with miR-124 by direct targeting (A) TCC-SUP and UM-UC-3 cells were transfected with si-NC/si-XIST, the expression of miR124 in response to XIST knockdown was determined using real-time PCR. (B) miR-124 mimics and miR-124 inhibitor was used to achieve miR124 overexpression and inhibition. The transfection efficiency was verified using real-time PCR. (C) A wtXIST as well as a mut-XIST luciferase reporter gene was constructed by mutating two predicted miR-124 binding sites in XIST. (D, E) The indicated luciferase reporter gene vectors were co-transfected into HEK293 cells with miR-124 mimics or inhibitor. The luciferase activity was then determined using dual luciferase assays. The data are showed as mean \pm SD of three independent experiments. ${ }^{*} \mathrm{P}<0.05,{ }^{* *} \mathrm{P}<0.01$.

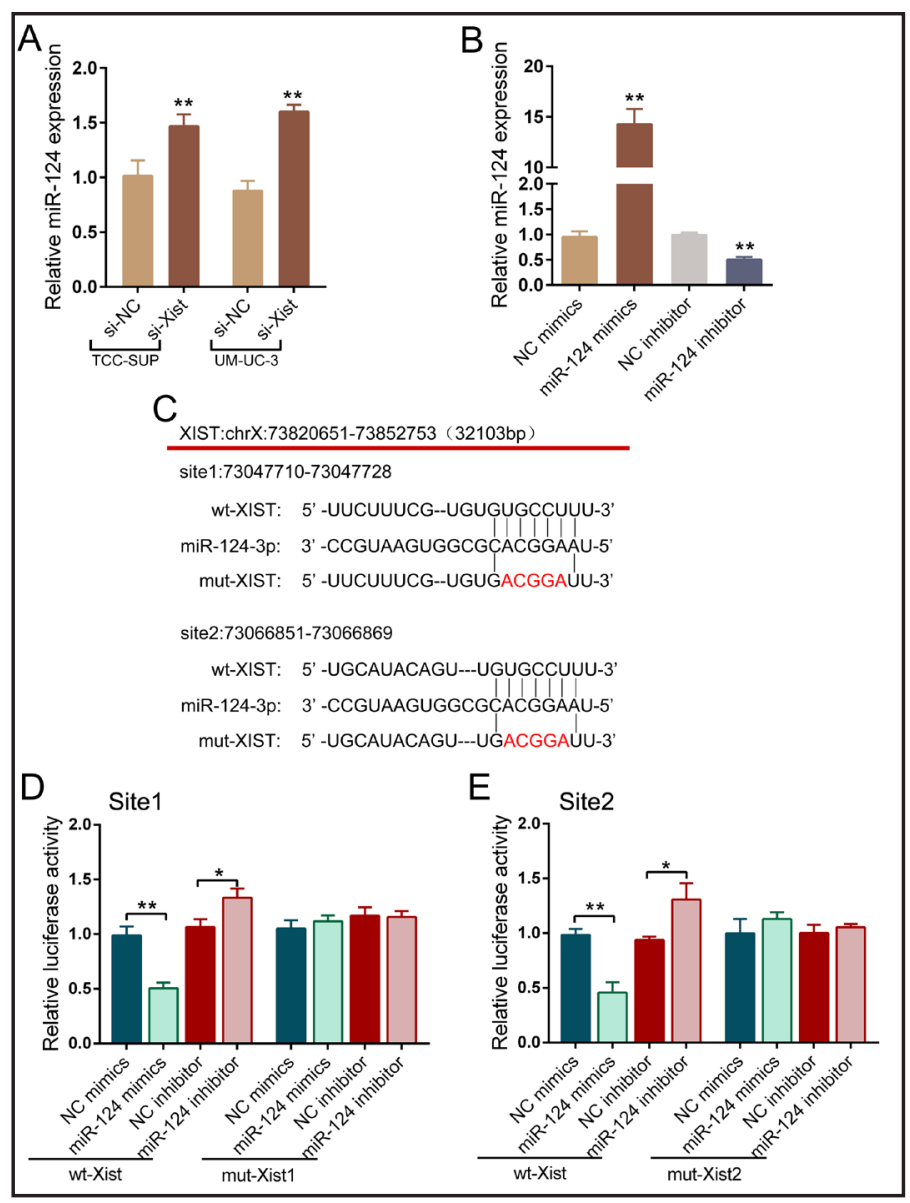

124 expression was significantly increased by si-XIST transfection (Fig. 3A), suggesting XIST indeed acted as a sponge of miR-124. Further, we investigated whether XIST binding to miR-124 to exert its function. MiR-124 mimics and miR-124 inhibitor were used to achieve miR-124 overexpression and inhibition, as verified using real-time PCR (Fig. 3B). We then constructed a wt-XIST luciferase reporter vector (wt-XIST), as well as a mut-XIST luciferase reporter vector (mut-XIST) by sequentially mutating two predicted miR-124 binding sites in XIST (Fig. 3C). The indicated vectors were then co-transfected with miR-124 NC/ miR-124 mimics into HEK293 cells to confirm XIST direct binding to miR-124. The luciferase activity of the wt-XIST luciferase reporter vector was significantly reduced in miR-124 mimics transfected HEK293 cells, whereas amplified in miR-124 inhibitor-transfected HEK293 cells, compared to mimics NC groups (Fig. 3D, E). After mutation in the predicted binding site of miR-124 within XIST, the changes of luciferase activity were abolished (Fig. 3D, E). These data indicated that XIST acts as a sponge of miR-124 to inhibit its expression through direct binding to miR-124.

\section{XIST promoted AR expression through miR-124}

Given that XIST inhibits miR-124 by targeting, and that XIST positively correlated with AR in bladder cancer tissues, we next investigated whether XIST regulated AR expression through miR-124. It was predicted that miR-124 could bind to and target the AR 3' UTR by using TargetScan, miRanda and miRWalk. A wt- $A R$ 3' UTR luciferase reporter vector (wt$\mathrm{AR}$ ) was created to confirm this prediction, as well as a mut- $A R 3^{\prime}$ UTR luciferase reporter vector (mut-AR) by sequentially mutating the predicted $5 \mathrm{bp}$ miR-124 binding site in the $A R$ 3' UTR (Fig. 4A). The indicated vectors and miR-124 mimics or inhibitor were co-transfected into HEK293 cells. In miR-124 mimics transfected cells the luciferase activity of the AR 3' UTR luciferase reporter vector was significantly reduced while it was induced in miR-124 
Fig. 4. XIST promoted AR expression through miR-124 (A) A wt-AR 3' UTR luciferase reporter vector (wt-AR), as well as a mut-AR 3' UTR luciferase reporter vector (mut-AR) by sequentially mutating predicted miR-124 binding site in the AR 3' UTR was generated. (B) The indicated luciferase reporter gene vectors and miR-124 inhibitor or miR-124 mimics were cotransfected into HEK239 cells. The luciferase activity of the AR 3'UTR luciferase reporter vector was determined using dual luciferase assays. (C) si-Xist and miR-124 inhibitor were co-transfected into UM-UC-3 cells, the protein levels of AR, c-myc, p27, MMP13 and MMP9 were then monitored using Western blot assays. Protein level of GAPDH was used as an endogenous normalization. (D) and (E) The activities of MMP9 and MMP13 were determined by SensoLytes Plus $^{\text {TM }} 520$ MMP9 and MMP13 assay kits. The data are showed as mean \pm SD of three independent experiments. ${ }^{*} \mathrm{P}<0.05,{ }^{* *} \mathrm{P}<0.01$.

inhibitor transfected cells, compared to inhibitor NC group (Fig. 4B). Besides, mutation of the putative miR-124 binding site in the $A R$ 3' UTR abolished miR-124-mediated repression of $A R$ 3' UTR luciferase reporter activity (Fig. 4B).

Accumulating evidences revealed that c-myc is an AR target gene in bladder cancer and breast cancer $[22,23]$. p27 is a major regulator of proliferation which exhibits inhibitory activity by binding cyclin E/Cdk2 complexes [24]. While, as an oncogene, c-myc abrogates p27 function in proliferation [25]. MMPs are a family of enzymes that degrade extracellular matrix (ECM), which promote tumor growth and metastasis [26]. In bladder cancer, MMP9 is correlated with tumor stage [27]. Moreover, increased AR signals might enhance BC cell invasion via increasing MMP13 expression [28]. Therefore, we monitored the protein levels of AR and proliferation-related factors, c-myc and p27, metastasis-related factors, MMP9 and MMP13, in response to the combined effect of si-XIST and miR-124 inhibitor in UMUC-3 cells, which is more malignant compared with TCC-SUP cells. Results from Western blot showed that XIST knockdown significantly reduced the protein levels of AR, c-myc, MMP9 
Fig. 5. The correlation of miR-124 with XIST and AR (A) Expression of miR-124 in a large panel of 67 paired bladder cancer tissues and corresponding adjacent non-tumor tissues was determined using real-time PCR. The data are showed as mean \pm SD of three independent experiments. ${ }^{* *} \mathrm{P}<0.01$. (B, C) Spearman's rank relation analysis was performed to analyze the correlation between XIST and miR-124, between AR and miR-124.

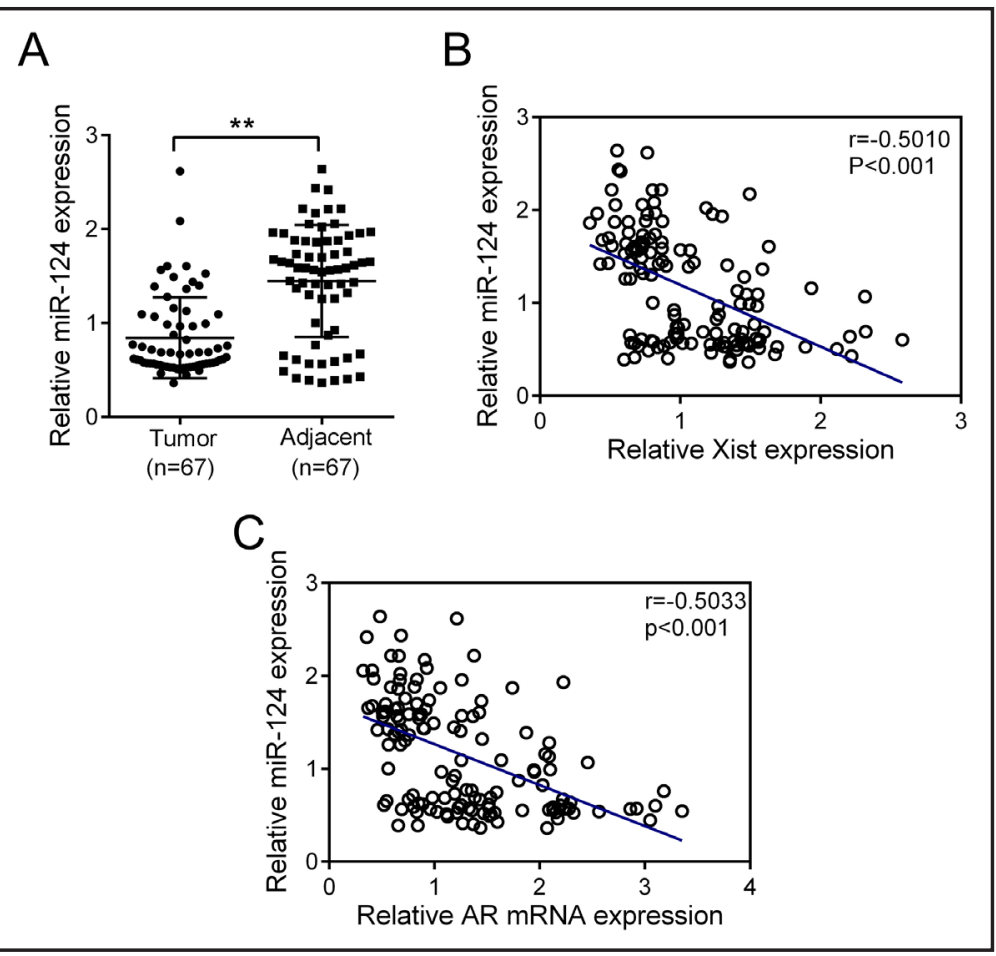

and MMP13, but increased the p27 levels; miR-124 inhibition significantly promoted the protein levels of AR, c-myc, MMP9 and MMP13, but decreased the p27 levels; the effect of miR-124 inhibition on the protein levels of the indicated factors could be partially restored by XIST knockdown (Fig. 4C). Moreover, the promote effect of miR-124 inhibition on MMP9 and MMP13 could be partially restored by XIST knockdown (Fig. 4D and E).Taken together, XIST regulates AR and proliferation and metastasis-related factors' expression most possibly through miR-124.

The correlation of miR-124 with XIST and AR

Expression of miR-124 was determined in a large panel of 67 paired bladder tissues and corresponding adjacent non-tumor tissues using real-time PCR. Results showed that miR-124 was significantly down-regulated in bladder cancer tissues (Fig. 5A). By performing Spearman's rank correlation analysis, we observed an inverse correlation between XIST and miR-124, an inverse correlation between AR and miR-124 (Fig. 5B, C).

\section{Discussion}

Bladder cancer is the most common urinary system malignancy that originates from the bladder mucosa [29]. Statistics have shown that morbidity for bladder cancer is high among the urinary system malignancies [30]. Previous evidence refers that bladder cancer occurs at any age both in male and female, but the incidence is three to four times greater in male than in female [31, 32]. AR was involved in the biology of various diseases, including bladder cancer [32,33]. Boorjian et al. revealed that AR is inversely correlated with bladder cancer pathologic tumor stage [34]. While, other studies confirmed that AR expression positively correlated with tumor stage and grade $[18,35]$. Hence, the overall contribution of AR expression to bladder cancer progression was controversial.

LncRNA XIST has been reported to be up-regulated in many cancers [15, 36-38]. In cell lines created from the tissue of a patient with collecting duct carcinoma of the kidney, the XIST gene was found to have an increase in copy number [39]. XIST gene amplification has also been detected in microsatellite-unstable sporadic human colorectal cancer tissue when compared to corresponding normal colorectal epithelium [40]. However, the exact role of 
XIST in bladder cancer remains unclear. In bladder cancer, AR is reported to promote cell proliferation and migration [41]. Initially we looked at XIST and AR expression in bladder cancer tissues and corresponding adjacent non-tumor tissues. XIST and AR were highly expressed in bladder cancer tissues compared with adjacent non-tumor tissues. They were positively correlated with each other in bladder cancer tissues. As well, in bladder cancer cell lines, XIST and AR showed higher expression level compared with a normal cell line. Moreover, high expression of XIST and AR was related to poorer TNM stage of bladder cancer.

To investigate the functional role of XIST and AR in bladder cancer, further experiments were arranged. After siRNA knock down of XIST, AR protein level in TCC-SUP and UM-UC-3 cells was significantly downregulated $(P<0.01)$; while the inhibitory effect of si-XIST on AR protein level could be partially restored by pCMV-AR transfection. Moreover, siRNA knock down of XIST could repress cell proliferation, invasion and migration of TCC-SUP and UMUC- 3 cells; the inhibitory effect of si-XIST on cell proliferation, invasion and migration could be partially restored by PCMV-AR transfection. These results suggested that XIST and AR functions as oncogenes in bladder cancer, consistent with the results from Fang et al. that XIST acts as an oncogene in non-small cell lung cancer [37], and the results from Zhang et al. that AR mediates proliferation of breast cancer cells [42]. Further experiments were performed to investigate the underlying mechanism by which XIST regulate AR expression and exerts its effect on bladder cancer cell proliferation, invasion and migration.

Alterations of miRNAs expression are implicated almost all fields of cancer biology, including cell growth, apoptosis, migration and/or invasion, and they can function as either tumor suppressors or oncogenes [43-45]. In this study, we focused on miR-124 because of its potential suppressive function in human malignances, including bladder cancer. Wang et al. indicated that miR-124 exerts tumor suppressive functions on the cell proliferation, motility and angiogenesis of bladder cancer by fine-tuning UHRF1 [21]. Zhang and colleagues reported that miR-124 retards bladder cancer growth by directly targeting CDK4 [46]. Moreover, lncRNAs can act as miRNA sponges, reducing their regulatory effect on mRNAs [13]. They could serve as ceRNAs to sponge miRNAs, thereby regulating gene expression. XIST functions as a miRNA sponge of miR-449a, which was a negative regulator of Bcl-2 [47]. XIST up-regulates the expression of miR-34a-5p targeted gene E2F3 through acting as a competitive 'sponge' of miR-34a-5p [48]. Here, we hypothesized that XIST might act as a competitive sponge of miR-124 to inhibit miR-124 expression. To verify this hypothesis, we evaluated miR-124 expression in response to XIST. In fact, in response to XIST knockdown, miR-124 expression was significantly up-regulated in TCC-SUP and UM-UC-3 cell lines. In addition, by using the luciferase reporter gene assays, we confirmed that XIST directly binds to miR-124 to inhibit its expression. These data indicated that XIST indeed serve as a sponge of miR-124.

The impact of specific miRNAs on cancer biology depends on their downstream targets $[49,50]$. Different prediction algorithms were used to predict gene targets for miR-124 so that to elucidate the underlying mechanisms involved in the miR-124-induced inhibition of bladder cancer growth invasion and migration. Given that XIST was positively correlated with AR, we next validated whether AR was the downstream gene of miR-124. As confirmed by luciferase reporter gene assays, miR-124 directly binds to the 3'UTR of AR. Previous studies reported that persistent AR activity in advanced disease regulates cell cycle activity, steroid biosynthesis and anabolic metabolism in conjunction with regulatory co-factors, such as the E2F family, c-myc and signal transducer and activator of transcription (STAT) transcription factors [51]. C-myc could repress the proliferation inhibitor p27 expression which is decreased in BC tissues [52]. MMPs are a family of enzymes that degrade extracellular matrix (ECM), which promote tumor growth and metastasis [26]. In bladder cancer, MMP9 is correlated with tumor stage [27]. Moreover, increased AR signals might enhance BC cell invasion via increasing MMP13 expression [28]. Here we further monitored the protein levels of AR, c-myc, p27, MMP13 and MMP9 in response to XIST knockdown and miR-124 inhibition. As showed by Western blot assays, the protein levels of AR, c-myc, MMP13 and MMP9 could be significantly down-regulated by si-XIST, while up-regulated by

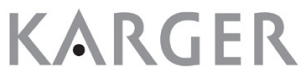




\section{Cellular Physiology Cell Physiol Biochem 2017;43:405-418 \begin{tabular}{ll|l} 
DOI: 10.1159/000480419 & $\begin{array}{l}\text { O } 2017 \text { The Author(s). Published by S. Karger AG, Basel } \\
\text { www.karger.com/cpb }\end{array}$ \\
\hline and Biochemistry
\end{tabular} \\ Xiong et al.: The XIST/miR-124/AR Axis Regulate Bladder Cancer Growth, Invasion and Migration}

miR-124 inhibition. In contrast, p27 protein levels could be significantly up-regulated by siXIST, while down-regulated by miR-124 inhibition. The inhibitory effect of si-XIST on these proteins could be partially restored by miR-124 inhibition. These data indicated that XIST might regulate bladder cancer cells' proliferation, invasion and migration through miR-124/ AR, and subsequently c-myc, p27, MMP13 and MMP9.

Moreover, miR-124 expression was down-regulated in bladder cancer tissues compared with corresponding adjacent non-tumor tissues which were consistent with previous report [53]. In bladder cancer tissues, miR-124 expression was inversely correlated with XIST and AR expression, respectively. These further indicated that, rescuing miR-124 expression to suppress the promotive effect of XIST on AR expression, and to inhibit AR expression, presented a promising strategy for regulation of bladder cancer cell proliferation and metastasis, thus to affect the progression of bladder cancer. In addition to the present study, dysfunctions of IncRNAs and miRNAs in bladder cancer have been frequently reported. Li et al. reported that UCA1 may serve as a natural molecular sponge to block the tumor suppressor role of miR-16; further, the 'seed region' of miR-16 directly binds to the 3'UTR of GLS2 mRNA and regulates GLS2 expression level. UCA1 regulates the expression of GLS2 through interfering with miR-16, and represses ROS formation in bladder cancer cells to promote bladder cancer progression [54]. SPRY4-IT1 positively regulated the expression of EZH2 through sponging miR-101-3p, and played an oncogenic role in bladder cancer progression [55]. Consistent with the previous studies, we also demonstrated that lncRNA acts as a sponge of miRNA, thus to regulate the downstream gene expression, which has been regarded as an essential mechanism of IncRNA-miRNA interaction [13]. By restoring the balance in this regulatory relationship, cancer progression can be affected, even improved.

\section{Conclusion}

In summary, it was identified that through AR, bladder cancer cell proliferation invasion and migration are promoted by XIST; these effects are due to the regulation of miR-124 by XIST. The XIST/miR-124/AR axis identified in the present study may play a key role in regulating bladder cancer cells proliferation, invasion and migration.

\section{Disclosure Statement}

The authors declare that there is no conflicts of interest regarding the publication of this paper.

\section{References}

1 Epstein JI, Allsbrook WC, Jr., Amin MB, Egevad LL: The 2005 International Society of Urological Pathology (ISUP) Consensus Conference on Gleason Grading of Prostatic Carcinoma. Am J Surg Pathol 2005;29:12281242.

-2 Witjes JA, Comperat E, Cowan NC, De Santis M, Gakis G, Lebret T, Ribal MJ, Van der Heijden AG, Sherif A: EAU guidelines on muscle-invasive and metastatic bladder cancer: summary of the 2013 guidelines. Eur Urol 2014;65:778-792.

-3 Sonpavde G, Goldman BH, Speights VO, Lerner SP, Wood DP, Vogelzang NJ, Trump DL, Natale RB, Grossman HB, Crawford ED: Quality of pathologic response and surgery correlate with survival for patients with completely resected bladder cancer after neoadjuvant chemotherapy. Cancer 2009;115:4104-4109.

4 Shen YJ, Kong ZL, Wan FN, Wang HK, Bian XJ, Gan HL, Wang CF, Ye DW: Downregulation of DAB2IP results in cell proliferation and invasion and contributes to unfavorable outcomes in bladder cancer. Cancer Sci 2014;105:704-712. 


\section{Cellular Physiology Cell Physiol Biochem 2017;43:405-418 \begin{tabular}{l|l|l|l|l}
\hline DOI: 10.1159/000480419 2017 The Author(s). Published by S. Karger AG, Basel & and Biochemistry
\end{tabular}

Xiong et al.: The XIST/miR-124/AR Axis Regulate Bladder Cancer Growth, Invasion and Migration

5 Li G, Song H, Wang J, Bao Y, Niu Y: Poor prognostic value of lymphovascular invasion for pT1 urothelial carcinoma with squamous differentiation in bladder cancer. Sci Rep 2016;6:27586.

6 Djebali S, Davis CA, Merkel A, Dobin A, Lassmann T, Mortazavi A, Tanzer A, Lagarde J, Lin W, Schlesinger F, Xue C, Marinov GK, Khatun J, Williams BA, Zaleski C, Rozowsky J, Roder M, Kokocinski F, Abdelhamid RF, Alioto T, Antoshechkin I, Baer MT, Bar NS, Batut P, Bell K, Bell I, Chakrabortty S, Chen X, Chrast J, Curado J, Derrien T, Drenkow J, Dumais E, Dumais J, Duttagupta R, Falconnet E, Fastuca M, Fejes-Toth K, Ferreira P, Foissac S, Fullwood MJ, Gao H, Gonzalez D, Gordon A, Gunawardena H, Howald C, Jha S, Johnson R, Kapranov P, King B, Kingswood C, Luo OJ, Park E, Persaud K, Preall JB, Ribeca P, Risk B, Robyr D, Sammeth M, Schaffer L, See LH, Shahab A, Skancke J, Suzuki AM, Takahashi H, Tilgner H, Trout D, Walters N, Wang H, Wrobel J, Yu Y, Ruan X, Hayashizaki Y, Harrow J, Gerstein M, Hubbard T, Reymond A, Antonarakis SE, Hannon G, Giddings MC, Ruan Y, Wold B, Carninci P, Guigo R, Gingeras TR: Landscape of transcription in human cells. Nature 2012;489:101-108.

-7 Martens-Uzunova ES, Bottcher R, Croce CM, Jenster G, Visakorpi T, Calin GA: Long noncoding RNA in prostate, bladder, and kidney cancer. Eur Urol 2014;65:1140-1151.

8 Ponting CP, Oliver PL, Reik W: Evolution and functions of long noncoding RNAs. Cell 2009;136:629-641.

-9 Zhou S, Wang J, Zhang Z: An emerging understanding of long noncoding RNAs in kidney cancer. J Cancer Res Clin Oncol 2014;140:1989-1995.

10 Yao Y, Ma J, Xue Y, Wang P, Li Z, Liu J, Chen L, Xi Z, Teng H, Wang Z: Knockdown of long non-coding RNA XIST exerts tumor-suppressive functions in human glioblastoma stem cells by up-regulating miR-152. Cancer Lett 2015;359:75-86.

11 Tantai J, Hu D, Yang Y, Geng J: Combined identification of long non-coding RNA XIST and HIF1A-AS1 in serum as an effective screening for non-small cell lung cancer. Int J Clin Exp Pathol 2015;8:7887-7895.

12 Ma L, Zhou Y, Luo X, Gao H, Deng X, Jiang Y: Long non-coding RNA XIST promotes cell growth and invasion through regulating miR-497/MACC1 axis in gastric cancer. Oncotarget 2016;8:4125-4135.

13 Paraskevopoulou MD, Hatzigeorgiou AG: Analyzing MiRNA-LncRNA Interactions. Methods Mol Biol 2016;1402:271-286.

14 Wu Z, Liu X, Liu L, Deng H, Zhang J, Xu Q, Cen B, Ji A: Regulation of lncRNA expression. Cell Mol Biol Lett 2014;19:561-575.

15 Chen DL, Ju HQ Lu YX, Chen LZ, Zeng ZL, Zhang DS, Luo HY, Wang F, Qiu MZ, Wang DS, Xu DZ, Zhou ZW, Pelicano H, Huang P, Xie D, Wang FH, Li YH, Xu RH: Long non-coding RNA XIST regulates gastric cancer progression by acting as a molecular sponge of miR-101 to modulate EZH2 expression. J Exp Clin Cancer Res 2016;35:142.

16 Zhuang LK, Yang YT, Ma X, Han B, Wang ZS, Zhao QY, Wu LQ Qu ZQ: MicroRNA-92b promotes hepatocellular carcinoma progression by targeting Smad7 and is mediated by long non-coding RNA XIST. Cell Death Dis 2016; 7:e2203.

17 Lombard AP, Mudryj M: The emerging role of the androgen receptor in bladder cancer. Endocrin Rel Cancer $2015 ; 22$

18 Mashhadi R, Pourmand G, Kosari F, Mehrsai A, Salem S, Pourmand MR, Alatab S, Khonsari M, Heydari F, Beladi L: Role of steroid hormone receptors in formation and progression of bladder carcinoma: a casecontrol study. Urology J 2014;11:1968-1973.

19 Salmena L, Poliseno L, Tay Y, Kats L, Pandolfi PP: A ceRNA hypothesis: the Rosetta Stone of a hidden RNA language? Cell 2011;146:353-358.

20 Cheng Y, Li Y, Nian Y, Liu D, Dai F, Zhang J: STAT3 is involved in miR-124-mediated suppressive effects on esophageal cancer cells. BMC Cancer 2015;15:306.

21 Wang X, Wu Q, Xu B, Wang P, Fan W, Cai Y, Gu X, Meng F: MiR-124 exerts tumor suppressive functions on the cell proliferation, motility and angiogenesis of bladder cancer by fine-tuning UHRF1. FEBS J 2015;282:4376-4388.

22 Gao L, Schwartzman J, Gibbs A, Lisac R, Kleinschmidt R, Wilmot B, Bottomly D, Coleman I, Nelson P, Mcweeney S: Androgen Receptor Promotes Ligand-Independent Prostate Cancer Progression through c-Myc Upregulation. Plos One 2013;8:e63563.

-23 Shen H, Lin Z, Feng X, Cong X, Li C, Yun N: Lin28A activates androgen receptor via regulation of c-myc and promotes malignancy of ER-/Her2+ breast cancer. Oncotarget 2016;7:60407-60418. 


\section{Cellular Physiology Cell Physiol Biochem 2017;43:405-418 \begin{tabular}{l|l|l|l|l}
\hline DOI: 10.1159/000480419 & $\begin{array}{l}\text { C) } 2017 \text { The Author(s). Published by S. Karger AG, Basel } \\
\text { www.karger.com/cpb }\end{array}$ \\
\hline
\end{tabular}

Xiong et al.: The XIST/miR-124/AR Axis Regulate Bladder Cancer Growth, Invasion and Migration

24 Prasad SB, Yadav SS, Das M, Modi A, Kumari S, Pandey LK, Singh S, Pradhan S, Narayan G: PI3K/AKT pathway-mediated regulation of p27 Kip1 is associated with cell cycle arrest and apoptosis in cervical cancer. Cell Oncol 2015;38:215-225.

-25 Griffin NI, Sharma G, Zhao X, Mirza S, Srivastava S, Dave BJ, Aleskandarany M, Rakha E, Mohibi S, Band H: ADA3 regulates normal and tumor mammary epithelial cell proliferation through c-MYC. Breast Cancer Res Bcr 2016;18:113.

-26 Wang Q, Diao X, Sun J, Chen Z: Regulation of VEGF, MMP-9 and metastasis by CXCR4 in a prostate cancer cell line. Cell Biol Inter 2011;35:897-904.

-27 Nutt JE, Durkan GC, Mellon JK, Lunec J: Matrix metalloproteinases (MMPs) in bladder cancer: the induction of MMP9 by epidermal growth factor and its detection in urine. Bju Inter 2003;91:99-104.

28 Lin C, Lin W, Yeh S, Li L, Chang C: Infiltrating neutrophils increase bladder cancer cell invasion via modulation of androgen receptor (AR)/MMP13 signals. Oncotarget 2015;6:43081-43089.

29 Schorey JS, Bhatnagar S: Exosome function: from tumor immunology to pathogen biology. Traffic 2008;9:871-881.

30 Ward E, DeSantis C, Robbins A, Kohler B, Jemal A: Childhood and adolescent cancer statistics, 2014. CA Cancer J Clin 2014;64:83-103.

31 Gakis G, Efstathiou J, Lerner SP, Cookson MS, Keegan KA, Guru KA, Shipley WU, Heidenreich A, Schoenberg MP, Sagaloswky AI, Soloway MS, Stenzl A: ICUD-EAU International Consultation on Bladder Cancer 2012: Radical cystectomy and bladder preservation for muscle-invasive urothelial carcinoma of the bladder. Eur Urol 2013;63:45-57.

32 Dobruch J, Daneshmand S, Fisch M, Lotan Y, Noon AP, Resnick MJ, Shariat SF, Zlotta AR, Boorjian SA: Gender and Bladder Cancer: A Collaborative Review of Etiology, Biology, and Outcomes. Eur Urol 2016;69:300-310.

-33 Gelmann EP: Molecular biology of the androgen receptor. J Clin Oncol 2002;20:3001-3015.

-34 Boorjian S, Ugras S, Mongan NP, Gudas LJ, You X, Tickoo SK, Scherr DS: Androgen receptor expression is inversely correlated with pathologic tumor stage in bladder cancer. Urology 2004;64:383-388.

35 Miyamoto H, Yao JL, Chaux A, Zheng Y, Hsu I, Izumi K, Chang C, Messing EM, Netto GJ, Yeh S: Expression of androgen and oestrogen receptors and its prognostic significance in urothelial neoplasm of the urinary bladder. BJU Int 2012;109:1716-1726.

-36 Schouten PC, Vollebergh MA, Opdam M, Jonkers M, Loden M, Wesseling J, Hauptmann M, Linn SC: High XIST and Low 53BP1 Expression Predict Poor Outcome after High-Dose Alkylating Chemotherapy in Patients with a BRCA1-like Breast Cancer. Mol Cancer Ther 2016;15:190-198.

37 Fang J, Sun CC, Gong C: Long noncoding RNA XIST acts as an oncogene in non-small cell lung cancer by epigenetically repressing KLF2 expression. Biochem Biophys Res Commun 2016;478:811-817.

38 Wang H, Shen Q, Zhang X, Yang C, Cui S, Sun Y, Wang L, Fan X, Xu S: The Long Non-Coding RNA XIST Controls Non-Small Cell Lung Cancer Proliferation and Invasion by Modulating miR-186-5p. Cell Physiol Biochem 2017;41:2221.

39 Wu ZS, Lee JH, Kwon JA, Kim SH, Han SH, An JS, Lee ES, Park HR, Kim YS: Genetic alterations and chemosensitivity profile in newly established human renal collecting duct carcinoma cell lines. BJU Int 2009;103:1721-1728.

40 Lassmann S, Weis R, Makowiec F, Roth J, Danciu M, Hopt U, Werner M: Array CGH identifies distinct DNA copy number profiles of oncogenes and tumor suppressor genes in chromosomal- and microsatelliteunstable sporadic colorectal carcinomas. J Mol Med (Berl) 2007;85:293-304.

-41 Ding G, Yu S, Cheng S, Li G, Yu Y: Androgen receptor (AR) promotes male bladder cancer cell proliferation and migration via regulating CD24 and VEGF. Am J Transl Res 2016;8:578-587.

42 Zhang W, Luo J, Yang F, Wang Y, Yin Y, Strom A, Gustafsson JA, Guan X: BRCA1 inhibits AR-mediated proliferation of breast cancer cells through the activation of SIRT1. Sci Rep 2016;6:22034.

-43 Hwang HW, Mendell JT: MicroRNAs in cell proliferation, cell death, and tumorigenesis. Br J Cancer 2007;96 Suppl:R40-44.

44 Zhi Y, Pan J, Shen W, He P, Zheng J, Zhou X, Lu G, Chen Z, Zhou Z: Ginkgolide B Inhibits Human Bladder Cancer Cell Migration and Invasion Through MicroRNA-223-3p. Cell Physiol Biochem 2016;39:1787.

45 Sun DK, Wang JM, Zhang P, Wang YQ: MicroRNA-138 Regulates Metastatic Potential of Bladder Cancer Through ZEB2. Cell Physiol Biochem 2015;37:2366.

46 Zhang T, Wang J, Zhai X, Li H, Li C, Chang J: MiR-124 retards bladder cancer growth by directly targeting CDK4. Acta Biochim Biophys Sin (Shanghai) 2014;46:1072-1079. 


\section{Cellular Physiology Cell Physiol Biochem 2017;43:405-418}

\begin{tabular}{ll|l} 
and Biochemistry & $\begin{array}{l}\text { DOI: 10.1159/000480419 } \\
\text { Published online: September 01, } 2017\end{array}$ & $\begin{array}{l}\text { O 2017 The Author(s). Published by S. Karger AG, Basel } \\
\text { www.karger.com/cpb }\end{array}$ \\
\cline { 2 - 3 }
\end{tabular}

Xiong et al.: The XIST/miR-124/AR Axis Regulate Bladder Cancer Growth, Invasion and Migration

47 Zhang YL, Li XB, Hou YX, Fang NZ, You JC, Zhou QH: The IncRNA XIST exhibits oncogenic properties via regulation of miR-449a and Bcl-2 in human non-small cell lung cancerThis article has been corrected since Advanced Online Publication, and an erratum is also printed in this issue. Acta Pharmacol Sin 2017;38:371381.

48 Song P, Ye LF, Zhang C, Peng T, Zhou XH: Long non-coding RNA XIST exerts oncogenic functions in human nasopharyngeal carcinoma by targeting miR-34a-5p. Gene 2016;592:8-14.

-49 Profumo V, Doldi V, Gandellini P, Zaffaroni N: Targeting microRNAs to withstand cancer metastasis. Methods Mol Biol 2015;1218:415-437.

-50 Chen J, Sun D, Chu H, Gong Z, Zhang C, Gong B, Li Y, Li N, Jiang L: Screening of differential microRNA expression in gastric signet ring cell carcinoma and gastric adenocarcinoma and target gene prediction. Oncol Rep 2015;33:2963-2971.

51 Lamb AD, Massie CE, Neal DE: The transcriptional programme of the androgen receptor (AR) in prostate cancer. BJU Int 2014;113:358-366.

-52 Romics I, Bánfi G, Székely E, Krenács T, Szende B: Expression of p21 waf1/cip1 , p27 kip1 ,p63 and Androgen Receptor in Low and High Gleason Score Prostate Cancer. Pathol Oncol Res 2008;14:307-311.

53 Wang X, Wu Q, Xu B, Wang P, Fan W, Ying C, Gu X, Meng F: miR-124 exerts tumor suppressive functions on the cell proliferation, motility and angiogenesis of bladder cancer by fine-tuning UHRF1. Febs J 2015;282:4376-4388.

54 Li HJ, Li X, Pang H, Pan JJ, Xie XJ, Chen W: Long non-coding RNA UCA1 promotes glutamine metabolism by targeting miR-16 in human bladder cancer. Jpn J Clin Oncol 2015;45:1055-1063.

55 Liu D, Li Y, Luo G, Xiao X, Tao D, Wu X, Wang M, Huang C, Wang L, Zeng F, Jiang G: LncRNA SPRY4-IT1 sponges miR-101-3p to promote proliferation and metastasis of bladder cancer cells through up-regulating EZH2. Cancer Lett 2017;388:281-291. 